\title{
Quark and Chromon Model and Glueball-Quarkonium Mixing in QCD
}

\author{
Y. M. Cho ${ }^{1,2,3, a}$ \\ ${ }^{1}$ Administration Building 310-4, Konkuk University, Seoul 143-701, Korea \\ ${ }^{2}$ Center for Quantum Spacetime, Sogang University, Seoul 04107, Korea \\ ${ }^{3}$ School of Physics and Astronomy, Seoul National University, Seoul 151-747, Korea
}

\begin{abstract}
The existence of two types of gluons in QCD, the color neutral neurons and colored chromons, implies that the chromons can be the constituent of hadrons. This generalizes the quark model to the quark and chromon model. We discuss the glueballquarkonium mixing in QCD based on the quark and chromon model. We present the neumerical analysis of glueball-quarkonium mixing below $2 \mathrm{GeV}$ in $0^{++}, 2^{++}$, and $0^{-+}$ channels, and predict the relative branching ratios of the radiative decay of $\psi$ to the physical states.
\end{abstract}

\section{Introduction}

An important issues in hadron spectroscopy is the identification of glueballs. The search for the glueballs has a long history [1,2]. The general wisdom is that QCD must have the glueballs made of gluons, and several models of glueballs have been proposed [3, 4]. Moreover, the lattice QCD has been able to estimate the mass of the low-lying glueballs [5,6]. But experimentally, it has been very difficult to pinpoint the glueballs, in spite of the fact that Particle Data Group (PDG) has accumulated large number of hadronic particles which do not seem to fit to the simple quark model [7].

The search for the glueballs has not been so successful for two reasons [8,9]. First, theoretically there has been no clear picture of the glueballs, although there have been many proposals of the glueball model. This makes it difficult to predict what kind of glueballs we can actually expect. To see this let us consider the two leading models of glueballs, the bag model and the constituent model. Both have critical defects.

The bag model proposes to identify the glueballs as the confined gluon fields in a bag [3]. According to this model the glueballs are made of gluon field (i.e., infinite number of gluons), so that there are no constituent gluons (a finite number of gluons) which make up the glueballs. The problem with this picture is that (if this picture is correct) we should have similar electromagnetic "photoballs" in

\footnotetext{
ae-mail: ymcho7@konkuk.ac.kr
} 
QED made of the electromagnetic fields confined in a bag. But experimentally we have no such states in QED.

On the other hand in the constituent model the glueballs are identified as the bound states of a finite number of the color octet "constituent gluons" [4]. Intuitively this model looks very attractive. But obviously we need the gluon field (i.e., infinite number of gluons) to bind them, so that we need an infinite number of binding gluons as well as a finite number of constituent gluons to make a glueball. Unfortunately, this model does not tell what is the difference between the constituent gluons and the binding gluons.

The other reason is that it is not clear how to identify the glueballs experimentally. This is partly because the glueballs could mix with the quarkoniums, so that we must take care of the possible mixing to identify the glueballs experimentally $[4,8,9]$. To do that, of course, we need a clear picture of glueballs. This is why we have very few candidates of the glueballs so far, compared to huge hadron spectrum made of quarks listed by PDG.

This makes the search for the glueballs an urgent issue in QCD. Indeed, one of the main purpose of the Jefferson Lab $12 \mathrm{GeV}$ upgrading is to search for the glueballs [10]. To have a successful identification of glueballs we must understand the dynamics of QCD more clearly. The Abelian decomposition simplifies the QCD dynamics greatly and could resolve the above difficulties [11, 12]. The purpose of this talk is to discuss the glueball-quarkonium mixing based on the Abelian decomposition.

The Abelian decomposition has many advatages[11, 12]. It decomposes the QCD gauge potential to the color neutral restricted potential and the colored valence potential gauge independently. This tells that in QCD there are two types of gluons which play different roles. The restricted potential describes the binding gluons (the neurons) which bind the colored source, while the valence potential describes the valence gluons (the chromons) which become the colored source of QCD. This allows us to view QCD as the restricted QCD (RCD) made of the neurons which has the chromons as the colored source.

Moreover, it decomposes the restricted potential further to the non-topological Maxwell part which describes the naive Abelian gluons and topological Dirac part which describes the non-Abelian monopoles gauge independently. This allows us to study the role of the monopole gauge independently, and to show that it is the monopole which confines the colored objects. In fact implementing the Abelian decomposition on lattice, we can calculate the contribution of the Wilson loop with the full ptential, the restricted potential, and the monopole potential separately, and show that the monopole potential is responsible for the confining force [13, 14].

Furthermore, with the Abelian decomposition we can calculate the QCD effective action in the presence of the monopole background and show that the true vacuum of QCD is given by the monopole, more precisely the monopole-antimonopole pair condensation $[15,16]$. This tells that it is the monopole condensation which generates the mass gap and the confinement in QCD.

But what is most important for our purpose is that it provides a clear picture of glueball which allows us to identify them experimentally. This is because the chromons, after the confinement sets in, play the role of the constituent gluons while the neurons becomes the binding gluons. So we can construct the glueballs with a finite number of chromons. This generalizes the quark model to the quark and chromon model which provides a totally new picture of hadrons [12, 17]. In particular, this provides a clear picture of glueballs and their mixing with quarkoniums. 
To understand this consider the atoms in QED. Obviously we have photons as well as electrons and nucleons in it, but only the electrons (and nucleons) become the constituents because only they determine the atomic structure of the atoms in the periodic table. The photons play no role in the atomic structure. They are there as the electromagnetic field to provide the binding, not as the constituent of the atoms.

Exactly the same way we have quarks and gluons in hadrons. But again the gluons inside the proton does not play any role in the baryonic structure of the proton. This means that they must be the "binding" gluons, not the "constituent" gluons, which (just like the photons in atoms) provide only the binding of the quarks in the proton. If so, what is the constituent gluons, and how can we distinguish them from the binding gluons? The constituent model can not provide the answer.

The Abelian decomposition naturally provides the answer. It tells that there are indeed two types of gluons, the binding gluons called the neurons and the valence gluons called the chromons, and only the chromons can be treated as the constituent gluons [11,12]. And the gluons in nucleons are the neurons, not the chromons, because only three constituent quarks characterize the baryonic structure of nucleons. This tells that the neurons can not be the constituent of hadrons. As importantly this tells that the chromons, just like the quarks, become a constituent of the hadrons. This naturally generallizes the quark model and leads us to the quark and chromon model of hadrons.

In principle we can construct an infinite number of glueballs with chromons. So one might worry that experimentally we have few candidates of them. Our model could explain why this is so. First, in our model the glueballs made of chromons have an intrinsic unstability [17]. So they have broad widths, and this is in addition to the normal hadronic decay width. This means that they have a relatively short life-time. So only the low-lying glueballs could actually be observed experimentally. This is because the chromons, unlike the quarks, tend to annihilate each other in the chromo-electric background. This must be contrasted with quarks, which remain stable inside the hadrons.

This is closely related to the asymptotic freedom (anti-screening) of gluons. It is well known that in QED the strong electric background tends to generate the pair creation of electrons, which makes the charge screening $[18,19]$. But in QCD gluons and quarks play opposite roles in the asymptotic freedom. The quarks enhance the screening while the gluons diminish it to generate the anti-screening [20]. In fact in the persence of a chromo-electric background the chromon loop generates a negative imaginary part but the quark loop generates a positive imaginary part in the QCD effective action. This tells that the chromo-electric field tends to generate the pair creation of the quarks but the pair annihilation of the chromons [15, 16, 21, 22].

Second, in our model the glueballs inevitably mix with quarkoniums, so that in general they do not appear as mass eigenstates. So, to identify the glueballs, we have to consider the possible mixing with the quarkoniums. This makes the experimental identification of glueballs a nontrivial matter. This is why there are few candidates of glueballs. In this talk we provide the numerical analysis of the glueball-quarkonium mixing to help identify the physical content of these particles without ambiguity.

\section{Neurons and Chromons: A Review}

It is well known that QCD can be understood as the extended QCD (ECD), namely RCD made of the binding gluons which has the valence gluons as colored source [11, 12]. This follows from the Abelian decomposition of QCD which decomposes the gauge potential to the color neutral restricted 


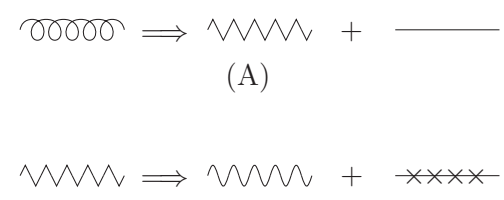

(B)

Figure 1. The Abelian decomposition of the gluons. The gluon is decomposed to the binding gluon (kinked line) and the valence gluon (straight line) in (A), and the binding gluon is further decomposed to the Maxwell part (wiggly line) and Dirac part (spiked line) in (B).

part made of the non-topological Maxwell potential and the topological Dirac potential and the gauge covariant colored valence part gauge independently.

We can summarize the Abelian decomposition graphically. This is shown in Fig. 1, where the gluons are decomposed to the neurons and the chromons in (A), and the neurons are decomposed further to the non-topological Maxwell part and the topological Dirac part in (B).

The Abelian decomposition does not change QCD. However, it refines QCD and simplifies the QCD dynamics greatly. This is because it decomposes the gluons to neurons and chromons which play totally different role [15-17]. This is shown graphically in Fig.2.

In (A) the three-point QCD gluon vertex is decomposed to two vertices made of one neuron and two chromons and three chromons. In (B) the four-point gluon vertex is decomposed to three vertices made of one neuron and three chromons, two neurons and two chromons, and four chromons. In (C) the quark-gluon vertex is decomposed to the quark-neuron vertex and quark-chromon vertex. Notice that three-point vertex made of three neurons or two neurons and one chromon, and four-point vertex made of three or four neurons are forbidden by the conservation of color. Without the Abelian decomposition this refinemet would have been impossible because all gluons are treated on equal footing.

We emphasize that the neurons are expressed by the wiggly lines (Maxwell part). This is because, although the neurons in the broad sense include the monopole potential (Dirac part), it is represented only by the Maxwell part after the confinement sets in. So, in the perturbative regime (inside the hadrons) only the Maxwell part contributes to the Feynman diagrams.

\section{Glueballs in the Quark and Chromon Model}

In a preceeding paper we have outlined how we can construct the glueballs from chromons [17]. The general picture of the glueballs in our model is very similar to the glueballs in the constituent model, so that we can construct color singlet glueballs with $g \bar{g}$ or $g g g$ [4]. The difference, of course, is that in our case only the six chromons become the constituent gluons because the other two gluons become the binding gluons (the neurons). So our glueballs are actually the chromoballs.

In the constituent gluon model the gluons are treated as a color octet. So one might wonder how the six chromons could form a color octet. To answer this we have to keep in mind that the chromons and neurons are defined only after the Abelian decomposition, after selecting the Abelian directions. This is because the neurons are the Abelian part of gluons. But an important thing about the Abelian decomposition is that changing the signature of the Abelian directions does not change the Abelian 


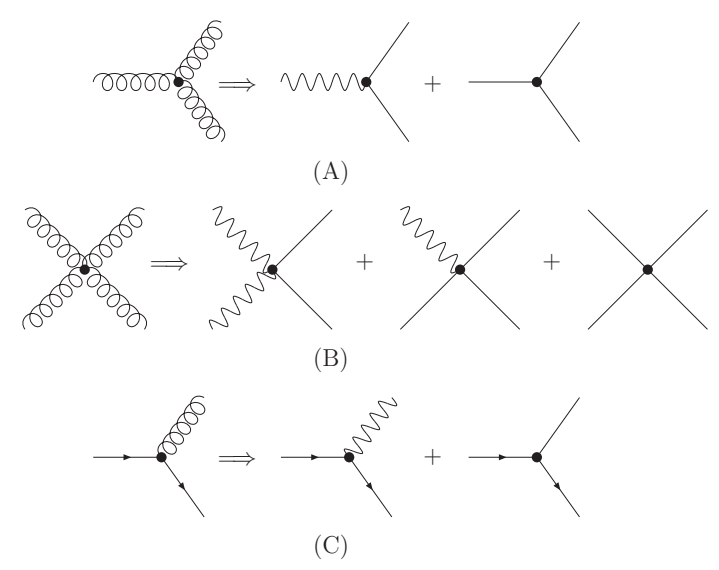

Figure 2. The decomposition of vertices in SU(3) QCD. The three and four point gluon vertices are decomposed in (A) and (B), and the quark gluon verteces are decomposed in (C). Notice that here (and in the followings) the neurons are representedby wiggly lines and the chromons are represented by straight lines.

decomposition, so that the Abelian decomposition is defined up to the residual symmetry called the color reflection invariance $[12,17]$. This means that, after the Abelian decomposition the color gauge symmetry is reduced to the descrete symmetry given by the color reflection group, and the gauge invariance translates to the color reflection invariance.

This observation tells that the chromons and neurons are defined only up to the color reflection under which they change the color. In other words, after the Abelian decomposition the color SU(3) is reduced to the descrete color reflection group. So the chromons and neurons should from a representation of the color reflection group, not the full color SU(3). And the color reflection invariant combinations of the chromons form the color singlet glueballs.

But obviously the color reflection group should form a subgroup of the original gauge group, so that for $\mathrm{SU}(\mathrm{n})$ it has an $n \times n$ matrix representation. For $\mathrm{SU}(2)$ it is made of 4 elements and for $\mathrm{SU}(3)$ it is made of 24 elements. The color reflection group has the Weyl group, the symmetry group of the root space of the original group, as a proper subgroup. The Weyl group of SU(n) is $S_{n}$, the symmetric group of degree $\mathrm{n}$ which is the permutation group of $\mathrm{n}$ elements.

So we can form the color reflection invariant (and Weyl invariant) combinations of $3 \times \overline{3}$ color siglets from $g \bar{g}$ and $3 \times 3 \times 3$ color singlets from $g g g$ chromons which obviously become singlets of the full color SU(3). Similarly we could have the color reflection invariant combinations of $3 \times \overline{3}$ color siglets from $q \bar{q}$ and $3 \times 3 \times 3$ color singlets from $q q q$ quarks.

We can in principle make the color reflection invariant combinations with two or three neurons. But the neurons are not likely to form bound states because they carry no color charge and interact among them very weakly. To see this we draw the possible Feynman diagrams for two neurons, two chromons, and two quarks in Fig. 3. The contrast between the neuron interaction and the chromon (and quark) interaction is striking. Again, this visualization would have been impossible without the Abelian decomposition. Obviously, two neuron binding is very much like two photon binding in QED. This strongly implies that the neurons do not form a bound state. Indeed, if the neurons form a bound state at all, it could be interpreted as a bound state of two quarkoniums. 


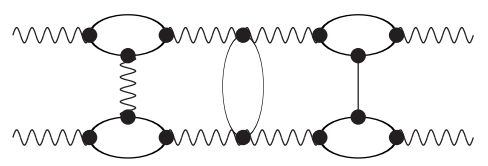

(A)

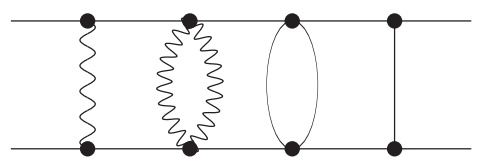

(B)

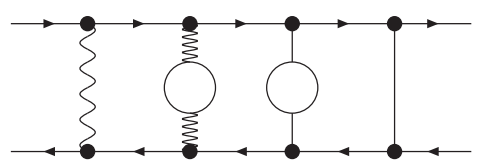

(C)

Figure 3. The possible Feynman diagrams of the neurons and chromons. Two neuron binding is shown in (A), two chromon binding is shown in (B), and two quark binding is shown in (C).

The above discussion tells that the chromon model is not the same as the gluon model where all gluons are treated as the constituent of hadrons. In particular, this tells that the color reflection group (and thus the Weyl group) should play the crucial role in all hadron spectroscopy. After the Abelian decomposition the color reflection invariance replaces the gauge invariance.

In the quark and chromon model it is natural to expect that the chromons (just like the quarks) acquire the constituent mass after the confinement sets in. So we may assume that in general the $g \bar{g}$ glueballs are lighter than the $g g g$ glueballs.

An important point of the chromon model is that the chromoballs have an intrinsic instability. As we have pointed out, in the chromoelectric background the chromons tend to annihilate each other. So inside the hadrons (in particular inside the chromoballs) where the chromoelectric flux is confined they become unstable. We can estimate the glueball partial decay width coming from this instability.

This implies that in general the glueballs (in particular excited ones) are expected to have very short lifetime, and become difficult to be observed. This could have been another reason why it was not easy to identify the glueball states experimentally.

\section{Glueball-Quarkonium Mixing: Numerical Analysis}

In the quark and chromon model it is clear that the mixing takes place not just between the quarkoniums and chromoballs but also between the $g \bar{g}$ and $g g g$ chromoballs, directly or through the virtual states made of neurons [17]. Obviously the mixing influences the $q \bar{q}$ octet-singlet mixing in the quark model. So we discuss the octet-singlet mixing first. 
Let

$$
\begin{gathered}
\langle u \bar{u}|H| u \bar{u}\rangle_{E x}=\langle d \bar{d}|H| d \bar{d}\rangle_{E x}=E, \\
\langle s \bar{s}|H| s \bar{s}\rangle_{E x}=E^{\prime}=E+\Delta, \\
\left.\left\langle q^{\prime} \overline{q^{\prime}}|H| q \bar{q}\right\rangle_{A n}=A, \quad \text { (for all } \mathrm{q}, \mathrm{q}^{\prime}\right) .
\end{gathered}
$$

Now with

$$
\begin{aligned}
|8\rangle & =\frac{|u \bar{u}\rangle+|d \bar{d}\rangle-2|s \bar{s}\rangle}{\sqrt{6}}, \\
|1\rangle & =\frac{|u \bar{u}\rangle+|d \bar{d}\rangle+|s \bar{s}\rangle}{\sqrt{3}},
\end{aligned}
$$

we may obtain the following mass matrix for the $q \bar{q}$ which describes the octet-singlet mixing,

$$
\begin{gathered}
M^{2}=\left(\begin{array}{cc}
\langle 8|H| 8\rangle & \langle 8|H| 1\rangle \\
\langle 1|H| 8\rangle & \langle 1|H| 1\rangle
\end{array}\right) \\
=\left(\begin{array}{cc}
E+\frac{2}{3} \Delta & -\frac{\sqrt{2}}{3} \Delta \\
-\frac{\sqrt{2}}{3} \Delta & E+\frac{1}{3} \Delta+3 A
\end{array}\right) .
\end{gathered}
$$

Notice that $\Delta$-term is responsible for the mixing. But we emphasize that this mixing among the quarks can not provide the correct octet-siglet mixing because the glueballs inevitably influence the quark octet-singlet mixing [17].

To proceed let us consider the $3 \times 3$ mixing of one lightest $g \bar{g}$ glueball $|G\rangle$ with the quark nonet for simplicity. In this case the mass matrix of the mixing can be written as

$$
M^{2}=\left(\begin{array}{ccc}
E+\frac{2}{3} \Delta & -\frac{\sqrt{2}}{3} \Delta & 0 \\
-\frac{\sqrt{2}}{3} \Delta & E+\frac{1}{3} \Delta+3 A & v \\
0 & v & G
\end{array}\right)
$$

where we can replace the parameter $G$ with the chromon constituent mass $\mu$ and put $G=4 \mu^{2}$.

We can diagonalize it with the mixing matrix which transforms the unphysical states $(|8\rangle,|1\rangle,|G\rangle)$ to the mass eigenstates $\left(\left|m_{1}\right\rangle,\left|m_{2}\right\rangle,\left|m_{3}\right\rangle\right)$. Clearly the mixing matrix has the information on the glueball and the quark octet and singlet contents of the physical states.

We can also generalize the mass matrix to the $4 \times 4$ mixing

$$
M^{2}=\left(\begin{array}{cccc}
E+\frac{2}{3} \Delta & -\frac{\sqrt{2}}{3} \Delta & 0 & 0 \\
-\frac{\sqrt{2}}{3} \Delta & E+\frac{1}{3} \Delta+3 A & v & v^{\prime} \\
0 & v & G & \epsilon \\
0 & v^{\prime} & \epsilon & G^{\prime}
\end{array}\right)
$$


to include one more $g \bar{g}$ or $g g g$ glueball state $\left|G^{\prime}\right\rangle$. In this case we may express $G$ and $G^{\prime}$ by the chromon mass $\mu$ and put $G=4 \mu^{2}$ and $G^{\prime}=4 \mu^{2}$ for $g \bar{g}$ or $G^{\prime}=9 \mu^{2}$ for $g g g$.

We emphasize the clarity of the mixing mechanism. All terms in (4) or (5) have clear physical meaning. For example we can draw the Feynman diagram which represents the parameter $v$ in (4), and could in principle calculate it theoretically.

With this we can predict the mass of the third state, calculate the quark and gluon contents of the physical states. From this we can estimate the relative branching ratios of the $\psi$ radiative decay to the physical states in each channel [17].

Let $\alpha_{i}$ be the parameters of the mixing matrix which determine the gluon content of physical states $\left|m_{i}\right\rangle$. We can predict the relative branching ratios of $\psi$ to $\gamma X$ decays among the physical states with $\alpha_{i}$, because this decay is the Okubo-Zweig-Iizuka (OZI) suppressed process which can only be made possible through the gluons. So, for the $\mathrm{S}$ wave decay (i.e., for $0^{++}$and $2^{++}$) we have

$$
R\left(\frac{\psi \rightarrow \gamma X_{k}}{\psi \rightarrow \gamma X_{i}}\right)=\left(\frac{\alpha_{k}}{\alpha_{i}}\right)^{2}\left(\frac{m_{\psi}^{2}-m_{k}^{2}}{m_{\psi}^{2}-m_{i}^{2}}\right)^{3}
$$

but for the $\mathrm{P}$ wave decay (i.e., for $0^{-+}$) we expect to have

$$
R\left(\frac{\psi \rightarrow \gamma X_{k}}{\psi \rightarrow \gamma X_{i}}\right)=\left(\frac{\alpha_{k}}{\alpha_{i}}\right)^{2}\left(\frac{m_{\psi}^{2}-m_{k}^{2}}{m_{\psi}^{2}-m_{i}^{2}}\right)^{5}
$$

where the last term is the kinematic phase space factor.

The above discussion shows that the mixing analysis is a crucial step for us to identify the glueballs. For the $3 \times 3$ mixing the mass matrix has five parameters, but we can fix $E$ and $\Delta$ from the $q \bar{q}$ flavor octet data. So we need three inputs to fix the mass matrix completely. There are different ways to fix them. One way is to choose two lowest mass eigenstates from PDG and treat $G$ (or the chromon mass $\mu$ ) as a free parameter, and find the best fit for $\mu$ which could explain the PDG data.

For the $4 \times 4$ mixing, the matrix has eight parameters, but we may fix two of them from the $q \bar{q}$ flavor octet data and express $G$ and $G^{\prime}$ by the chromon mass $\mu$. This reduces the number of the parameters to five. To do that we can choose the masses of three physical states as the input and vary the chromon mass $\mu$ as a free parameter, but we still need to fix one more parameter. for this purpose we may put $v^{\prime}=v$ for $g \bar{g}$ glueball $G^{\prime}$ or $v^{\prime}=3 / 2 v$ for $g g g$ glueball $G^{\prime}$, because $v$ and $v^{\prime}$ represent two and three gluon couplings to the iso-singlet $q \bar{q}$. With this to predict the mass of the fourth physical state. In the following we show how the glueballs can be identified in the quark and chromon model numerically.

\section{1 $0^{++}$channel}

In this channel PDG has five iso-singlet mesons, $f_{0}(500), f_{0}(980), f_{0}(1370), f_{0}(1500)$, and $f_{0}(1710)$ below $2 \mathrm{GeV}$ [7]. To discuss the mixing we have to choose two inputs $E$ and $\Delta$ first. Since PDG suggests that the isotriplet partner of the flavor octet in this channel is $a_{0}(1450)$, we may choose [17]

$$
\begin{gathered}
E=a_{0}^{2}, \quad a_{0}=a_{0}(1450), \\
\Delta=2\left(K^{2}-a_{0}^{2}\right), \quad K=K_{0}^{*}(1430) .
\end{gathered}
$$


Table 1. The numerical analysis of the $4 \times 4$ mixing in the $0^{++}$channel, with $f_{0}(980), f_{0}(1370), f_{0}(1500)$, and $f_{0}(1710)$ as the input. Here $a_{0}(1450)$ is identified as the isotriplet partner.

\begin{tabular}{ccccccccccccccccc}
\hline \hline$\mu$ & \multicolumn{4}{c}{$m_{1}=f_{0}(980)$} & \multicolumn{4}{c}{$m_{2}=f_{0}(1370)$} & \multicolumn{3}{c}{$m_{3}=f_{0}(1500)$} & \multicolumn{4}{c}{$m_{4}=f_{0}(1710)$} \\
& $u+d$ & $s$ & $G$ & $G^{\prime}$ & $u+d$ & $s$ & $G$ & $G^{\prime}$ & $u+d$ & $s$ & $G$ & $G^{\prime}$ & $u+d$ & $s$ & $G$ & $G^{\prime}$ \\
\hline 0.50 & 0.01 & 0.01 & 0.99 & 0.00 & 0.19 & 0.81 & 0.00 & 0.01 & 0.13 & 0.00 & 0.00 & 0.86 & 0.68 & 0.18 & 0.01 & 0.13 \\
\hline 0.52 & 0.03 & 0.03 & 0.94 & 0.00 & 0.18 & 0.80 & 0.01 & 0.01 & 0.17 & 0.01 & 0.02 & 0.81 & 0.62 & 0.17 & 0.04 & 0.17 \\
\hline 0.54 & 0.06 & 0.05 & 0.88 & 0.01 & 0.18 & 0.79 & 0.01 & 0.01 & 0.21 & 0.01 & 0.04 & 0.74 & 0.55 & 0.15 & 0.06 & 0.24 \\
\hline 0.56 & 0.09 & 0.08 & 0.82 & 0.01 & 0.18 & 0.79 & 0.02 & 0.01 & 0.26 & 0.01 & 0.08 & 0.65 & 0.47 & 0.13 & 0.08 & 0.33 \\
\hline 0.58 & 0.13 & 0.11 & 0.75 & 0.01 & 0.18 & 0.78 & 0.03 & 0.01 & 0.32 & 0.01 & 0.14 & 0.53 & 0.38 & 0.10 & 0.08 & 0.44 \\
\hline 0.60 & 0.17 & 0.14 & 0.67 & 0.02 & 0.17 & 0.77 & 0.05 & 0.01 & 0.38 & 0.01 & 0.22 & 0.39 & 0.27 & 0.07 & 0.07 & 0.58 \\
\hline \hline
\end{tabular}

\begin{tabular}{cccccccc}
\hline \hline$\mu$ & $R\left(m_{2} / m_{1}\right)$ & $R\left(m_{3} / m_{1}\right)$ & $R\left(m_{4} / m_{1}\right)$ & $\mathrm{A}$ & $v$ & $v^{\prime}$ & $\delta$ \\
\hline 0.50 & 0.01 & 0.54 & 0.06 & 0.25 & 0.19 & 0.24 & 1.35 \\
\hline 0.52 & 0.01 & 0.54 & 0.10 & 0.21 & 0.41 & 0.28 & 1.30 \\
\hline 0.54 & 0.02 & 0.54 & 0.16 & 0.17 & 0.53 & 0.32 & 1.26 \\
\hline 0.56 & 0.03 & 0.54 & 0.22 & 0.13 & 0.61 & 0.36 & 1.22 \\
\hline 0.58 & 0.04 & 0.54 & 0.31 & 0.07 & 0.66 & 0.40 & 1.20 \\
\hline 0.60 & 0.06 & 0.54 & 0.43 & 0.01 & 0.68 & 0.42 & 1.21 \\
\hline \hline
\end{tabular}

Table 2. The numerical analysis of the $4 \times 4$ mixing in the $0^{++}$channel, with $f_{0}(980), f_{0}(1370), f_{0}(1500)$, and $f_{0}(1710)$ as the input. Here $a_{0}(980)$ is identified as the isotriplet partner.

\begin{tabular}{|c|c|c|c|c|c|c|c|c|c|c|c|c|c|c|c|c|}
\hline \multirow[t]{2}{*}{$\mu$} & \multicolumn{4}{|c|}{$m_{1}=f_{0}(980)$} & \multicolumn{4}{|c|}{$m_{2}=f_{0}(1370)$} & \multicolumn{4}{|c|}{$m_{3}=f_{0}(1500)$} & \multicolumn{4}{|c|}{$m_{4}=f_{0}(1710)$} \\
\hline & $u+d$ & $s$ & $G$ & $G^{\prime}$ & $u+d$ & $s$ & $G$ & $G^{\prime}$ & $u+d$ & $s$ & $G$ & $G^{\prime}$ & $u+d$ & $s$ & $G$ & $G^{\prime}$ \\
\hline 0.67 & 0.86 & 0.00 & 0.14 & 0.01 & 0.13 & 0.04 & 0.83 & 0.04 & 0.01 & 0.01 & 0.00 & 0.98 & 0.01 & 0.96 & 0.03 & 0.01 \\
\hline 0.68 & 0.90 & 0.00 & 0.05 & 0.05 & 0.04 & 0.01 & 0.94 & 0.01 & 0.05 & 0.06 & 0.00 & 0.89 & 0.01 & 0.93 & 0.01 & 0.05 \\
\hline
\end{tabular}

\begin{tabular}{cccccccc}
\hline \hline$\mu$ & $R\left(m_{2} / m_{1}\right)$ & $R\left(m_{3} / m_{1}\right)$ & $R\left(m_{4} / m_{1}\right)$ & $\mathrm{A}$ & $v$ & $v^{\prime}$ & $\delta$ \\
\hline 0.67 & 4.17 & 4.21 & 0.11 & 0.08 & 0.40 & 0.13 & 0.47 \\
\hline 0.68 & 7.07 & 5.73 & 0.26 & 0.07 & 0.25 & 0.35 & 0.40 \\
\hline \hline
\end{tabular}

This looks somewhat strange because this implies that the $q \bar{q}$ state made of $u+d$ quark is heavier (or at least not lighter) than the $q \bar{q}$ state made of the $s$ quark.

So one could identify $a_{0}(980)$ as the isotriplet partner of the flavor octet. In this case we might choose

$$
\begin{gathered}
E=a_{0}^{2}, \quad a_{0}=a_{0}(980), \\
\Delta=2\left(K^{2}-a_{0}^{2}\right), \quad K=K_{0}^{*}(1430) .
\end{gathered}
$$

This seems natural because $a_{0}(980)$ is supposed to be made of $u$ and $d$ quarks lighter than $s$ quark.

Another point is the nature of $f_{0}(500)$. It is well known that this state does not fit to the quark model well, and has been suggested to be a molecular bound state of two mesons [7]. In our scheme this could be identified as the vacuum fluctuation mode of the monopole condensation [17]. Because of this we exclude this in this numerical analysis. 
So we consider the $4 \times 4$ mixing (5) with two chromoball states ${ }^{1} S_{0}$ and ${ }^{5} D_{0}$ made of two chromons $|G\rangle$ and $\left|G^{\prime}\right\rangle$. Diagonalizing the two chromoball mass matrix first, we may put

$$
\epsilon=0, \quad G=4 \mu^{2}, \quad G^{\prime}=G+\delta
$$

and consider the mixing of the two $q \bar{q}$ states with two chromoballs which have mass $\sqrt{G}$ and $\sqrt{G^{\prime}}$. This has seven parameters, but we can fix two with (8) or (9) and four with the four mass eigenstates $f_{0}(980), f_{0}(1370), f_{0}(1500)$, and $f_{0}(1710)$ as the input. With this we can diagonalize the mass matrix and find the physical contents of the mass eigenstates, treating the chromon mass $\mu$ as the free parameter.

Now, adopting the PDG view (8) we obtain Table 1, but with (9) we obtain Table 2. But the mathematical equations which we need to solve to diagonalize the mass matrix are very rigid which often have no solution, and this forces us to change the input data slightly to find the solutions. So here we have changed the four mass eigenstates to $990,1400,1505$, and $1722 \mathrm{MeVs}$ to obtain Table 1, and to 990, 1370, 1505, $1800 \mathrm{MeVs}$ to obtain Table 2.

The numerical result of Table 1 obtained with (8) suggests that $f_{0}(980)$ is predominantly the ${ }^{1} S_{0}$ chromoball state and $f_{0}(1370)$ is predominantly the $s \bar{s}$ state. But $f_{0}(1500)$ becomes largely the ${ }^{5} D_{0}$ chromoball state and $f_{0}(1710)$ becomes largely the $u \bar{u}+d \bar{d}$ state, although they have considerable mixing as the chromon mass increases to $600 \mathrm{MeV}$. But here the $u \bar{u}+d \bar{d}$ state remains heavier than the $s \bar{s}$ state, which again is due to the input (8).

Table 3. The numerical analysis of the $4 \times 4$ mixing in the $2^{++}$channel, with states $f_{2}(1270), f_{2}^{\prime}(1525), f_{2}(1950)$ as the input. The fourth state could be interpreted as $f_{2}(2010)$.

\begin{tabular}{|c|c|c|c|c|c|c|c|c|c|c|c|c|c|c|c|c|c|}
\hline \multirow[t]{2}{*}{$\mu$} & \multirow[t]{2}{*}{$m_{4}$} & \multicolumn{4}{|c|}{$m_{1}=f_{2}(1270)$} & \multicolumn{4}{|c|}{$m_{2}=f_{2}^{\prime}(1525)$} & \multicolumn{4}{|c|}{$\overline{m_{3}=f_{2}(1950)}$} & \multicolumn{4}{|c|}{$m_{4}$} \\
\hline & & $u+d$ & $s$ & $G$ & $G^{\prime}$ & $u+d$ & $s$ & $G$ & $G^{\prime}$ & $u+d$ & $s$ & $G$ & $G^{\prime}$ & $u+d$ & $s$ & $G$ & $G^{\prime}$ \\
\hline 0.90 & 2.86 & 0.80 & 0.01 & 0.19 & 0.00 & 0.06 & 0.85 & 0.09 & 0.00 & 0.14 & 0.14 & 0.7 & 0.00 & 0.00 & 0.00 & 0.00 & 0.99 \\
\hline 0.91 & 2.11 & 0.79 & 0.01 & 0.18 & 0.02 & 0.06 & 0.85 & 0.08 & 0.01 & 0.09 & 0.09 & 0.6 & 0.14 & 0.05 & 0.05 & 0.06 & 0.84 \\
\hline 0.92 & 2.07 & 0.79 & 0.01 & 0.18 & 0.02 & 0.06 & 0.85 & 0.08 & 0.01 & 0.05 & 0.05 & 0.5 & 0.36 & 0.10 & 0.09 & 0.21 & 0.61 \\
\hline 0.93 & 2.07 & 0.79 & 0.01 & 0.18 & 0.03 & 0.06 & 0.86 & 0.07 & 0.01 & 0.02 & 0.02 & 0.4 & 0.54 & 0.13 & 0.11 & 0.33 & 0.43 \\
\hline \multirow[t]{7}{*}{0.94} & 2.08 & 0.78 & 0.01 & 0.17 & 0.03 & 0.06 & 0.86 & 0.07 & 0.01 & 0.01 & 0.01 & 0.3 & 0.65 & 0.14 & 0.12 & 0.43 & 0.21 \\
\hline & & & & $\mu$ & $m_{4}$ & $R\left(m_{2} /\right.$ & & $R\left(m_{3} / r\right.$ & & $R\left(m_{4} / m_{1}\right)$ & & & $v$ & $\epsilon$ & & & \\
\hline & & & & 0.90 & 2.86 & 1.48 & & 0.03 & & 0.37 & & & 0.89 & 4.89 & & & \\
\hline & & & & 0.91 & 2.11 & 1.6 & & 1.23 & & 0.34 & & & 0.91 & 0.98 & & & \\
\hline & & & & 0.92 & 2.07 & 1.75 & & 1.20 & & 0.33 & & & 0.95 & 0.63 & & & \\
\hline & & & & 0.93 & 2.07 & 1.82 & & 1.10 & & 0.32 & & & 0.98 & 0.46 & & & \\
\hline & & & & 0.94 & 2.08 & 1.85 & & 1.01 & & 0.31 & & & 1.02 & 0.33 & & & \\
\hline
\end{tabular}

On the other hand, Table 2 obtained with (9) tells that $f_{0}(980)$ and $f_{0}(1710)$ are the $u \bar{u}+d \bar{d}$ and $s \bar{s}$ states, respectively. And $f_{0}(1370)$ and $f_{0}(1500)$ become the ${ }^{1} S_{0}$ and ${ }^{5} D_{0}$ chromoball states.

As for the $J / \psi$ radiative decay branching ratio, Table 1 shows that $R\left(f_{0}(1710) / f_{0}(1500)\right) \simeq 0.8$ when $\mu=0.60$, and Table 2 gives around 0.05 when $\mu=680 \mathrm{MeV}$. Clearly both are too small to explain the experimental value [7]. So we can not tell which is the isotriplet partner of the $0^{++}$ isosinglet state. 
Table 4. The numerical analysis of the $4 \times 4$ mixing in the $2^{++}$channel, with $f_{2}(1270), f_{2}^{\prime}(1525), f_{2}(1950)$, and $f_{2}(2010)$ as the input.

\begin{tabular}{cccccccccccccccccc}
\hline \hline$\mu$ & \multicolumn{4}{c}{$m_{1}=f_{2}(1270)$} & \multicolumn{4}{c}{$m_{2}=f_{2}^{\prime}(1525)$} & \multicolumn{4}{c}{$m_{3}=f_{2}(1950)$} & \multicolumn{4}{c}{$m_{4}=f_{2}(2010)$} \\
& $u+d$ & $s$ & $G$ & $G^{\prime}$ & $u+d$ & $s$ & $G$ & $G^{\prime}$ & $u+d$ & $s$ & $G$ & $G^{\prime}$ & $u+d$ & $s$ & $G$ & $G^{\prime}$ \\
\hline 0.90 & 0.80 & 0.01 & 0.19 & 0.00 & 0.06 & 0.85 & 0.09 & 0.00 & 0.13 & 0.14 & 0.72 & 0.01 & 0.01 & 0.00 & 0.01 & 0.99 \\
\hline 0.91 & 0.79 & 0.01 & 0.18 & 0.02 & 0.06 & 0.85 & 0.08 & 0.01 & 0.09 & 0.09 & 0.67 & 0.15 & 0.05 & 0.05 & 0.07 & 0.83 \\
\hline 0.92 & 0.79 & 0.01 & 0.17 & 0.03 & 0.06 & 0.85 & 0.07 & 0.01 & 0.06 & 0.06 & 0.61 & 0.27 & 0.09 & 0.08 & 0.14 & 0.69 \\
\hline 0.93 & 0.79 & 0.01 & 0.16 & 0.04 & 0.06 & 0.86 & 0.07 & 0.01 & 0.03 & 0.03 & 0.54 & 0.39 & 0.12 & 0.10 & 0.22 & 0.55 \\
\hline 0.94 & 0.78 & 0.01 & 0.16 & 0.04 & 0.06 & 0.86 & 0.07 & 0.02 & 0.01 & 0.01 & 0.44 & 0.53 & 0.14 & 0.12 & 0.33 & 0.41 \\
\hline \hline
\end{tabular}

\begin{tabular}{cccccccc}
\hline \hline$\mu$ & $R\left(m_{2} / m_{1}\right)$ & $R\left(m_{3} / m_{1}\right)$ & $R\left(m_{4} / m_{1}\right)$ & $\mathrm{A}$ & $v$ & $v^{\prime}$ & $\delta$ \\
\hline 0.90 & 1.49 & 1.43 & 0.37 & 0.11 & 0.89 & 0.14 & 1.16 \\
\hline 0.91 & 1.62 & 1.26 & 0.34 & 0.14 & 0.91 & 0.44 & 0.94 \\
\hline 0.92 & 1.71 & 1.13 & 0.33 & 0.15 & 0.93 & 0.56 & 0.75 \\
\hline 0.93 & 1.78 & 1.04 & 0.31 & 0.17 & 0.96 & 0.62 & 0.56 \\
\hline 0.94 & 1.82 & 0.98 & 0.31 & 0.18 & 0.99 & 0.62 & 0.39 \\
\hline \hline
\end{tabular}

\section{$4.22^{++}$channel}

In this channel we have three physical states below $2 \mathrm{GeV}, f_{2}(1270), f_{2}^{\prime}(1525)$, and $f_{2}(1950)$. On the other hand we have to keep in mind that there is the fourth state $f_{2}(2010)$ just above $2 \mathrm{GeV}$, which could better be included in the mixing. Another point is that PDG lists five more unestablished states, $f_{2}(1430), f_{2}(1565), f_{2}(1640), f_{2}(1810)$, and $f_{2}(1910)$, which could turn out to be real states. In this paper we will consider only the three plus $f_{2}(2010)$ established states in the mixing analysis, but the fact that there are so many unestablished $2^{++}$states implies that we have to be careful to analyse this channel.

We can do the $4 \times 4$ mixing with the three mass eigenstates below $2 \mathrm{GeV}$ and [17]

$$
\begin{gathered}
E=m^{2}\left(a_{2}\right), \quad a_{2}=a_{2}(1320), \\
\Delta=2\left(m^{2}\left(K^{*}\right)-m^{2}\left(a_{2}\right)\right), \quad K^{*}=K_{2}^{*}(1430),
\end{gathered}
$$

as the input, and try to predict the fourth state. The result is shown in Table 3. But here again we have changed the mass eigenvalues to 1275,1500 , and $1944 \mathrm{MeV}$ s to obtain the solutions. Remarkably it predicts that the mass of the fourth state is around $2100 \mathrm{MeV}$, which we can identify to be $f_{2}(2010)$.

In fact, since we have $f_{0}(2010)$ just above $2 \mathrm{GeV}$, it would be unfair to exclude this in the mixing. So we consider the $4 \times 4$ mixing with the four mass eigenstates and (11) as the input, and obtain Table 4. Here again we have changed the input masses a little, to $1275,1500,1944$, and $2100 \mathrm{MeVs}$, to find the solution.

Remarkably the result in Table 4 is very similar to the Table 3. Although the numbers are different, the general feature is the same. Here again $f_{2}(1270)$ becomes predominantly the $u \bar{u}+d \bar{d}$ state, $f_{2}^{\prime}(1525)$ becomes predominantly the $s \bar{s}$ state, and $f_{2}(1950)$ becomes predominantly a chromoball state. The only new thing is that $f_{2}(2010)$ becomes the second chromoball state, so that we can interpret $f_{2}(1950)$ and $f_{2}(2010)$ to be predominantly the ${ }^{5} S_{2}$ and ${ }^{1} D_{2}$ chromoballs.

The main difference between the two tables is the $J / \psi$ relative radiative decay ratio, which is very sensitive to the chromoball contents of the physical states. In Table 4 the radiative decay ratios turn 
out to be larger then the PDG values [7]. But we find that the ratios could be reduced to PDG values by changing the mass of $f_{2}^{\prime}(1525)$ to around $1490 \mathrm{MeV}$.

So all in all the mixing in the $2^{++}$channel seems to work fine, and the upshot of our mixing is that $f_{2}(1950)$ and $f_{0}(2010)$ are predominantly the chromoball states. On the other hand, it is good to remember that there are different suggestions in the literature. Clearly there have been claims that $f_{2}(1270)$ and $f_{2}^{\prime}(1525)$ are the $q \bar{q}$ states as PDG suggests $[8,9]$. Moreover, there have been assertions that they can be viewed as molecular states $[8,9]$.

Moreover, it is good to remember that here we have five unestablished states, $f_{2}(1430), f_{2}(1565)$, $f_{2}(1640), f_{2}(1810)$, and $f_{2}(1910)$ [7]. Some of them could turn out to be real states and make the mixing unreliable. So we need more analysis to understand the $2^{++}$mixing clearly.

\section{$4.30^{-+}$channel}

In this channel we have five established states below $2 \mathrm{GeV}, \eta(548), \eta^{\prime}(958), \eta(1295), \eta(1405)$, and $\eta(1475)$, and one unestablished state $\eta(1760)$. But since this channel needs a special attention because of the octet-singlet mixing, the U(1) problem, PCAC etc., we choose a slightly different approach and consider the $5 \times 5$ mixing matrix

$$
M^{2}=\left(\begin{array}{ccccc}
E+\frac{2}{3} \Delta & -\frac{\sqrt{2}}{3} \Delta & 0 & 0 & 0 \\
-\frac{\sqrt{2}}{3} \Delta & E+\frac{1}{3} \Delta+3 A & v_{1} & v_{2} & v_{3} \\
0 & v_{1} & G_{1} & 0 & 0 \\
0 & v_{2} & 0 & G_{2} & 0 \\
0 & v_{3} & 0 & 0 & G_{3}
\end{array}\right)
$$

which describes the mixing of two quarkonia and three chromoball states, two $g g$ and one $g g g$, below $2 \mathrm{GeV}$. This has nine parameters.

Now, we have [17]

$$
\begin{gathered}
E=m^{2}(\pi), \quad \pi=\pi(140), \\
\Delta=2\left(m^{2}(K)-m^{2}(\pi)\right), \quad K=K(498) .
\end{gathered}
$$

With this we chould choose five mass eigenvalues as the input. But here we choose a slightly different input. We choose four mass eigenstates, $\eta^{\prime}(958), \eta(1295), \eta(1405), \eta(1475)$, and the experimental value of $R\left(\eta(1475) / \eta^{\prime}(958)\right)=0.95$ in stead of $\eta(548)$. With this we could predict the mass of the fifth physical state. The reason is that, sincethe mathematical equations which we need to solve to diagonalize the mass matrix are very rigid, we could not find the solution when we use the five mass eigenstates as the inputs.

Assuming that $G_{3}$ is the $g g g$ chromoball we let

$$
G_{1}=4 \mu^{2}, \quad G_{2}=4 \mu^{2}+\delta, \quad G_{3}=9 \mu^{2}+\delta^{\prime},
$$

and obtain Table 5 using (13). The result shows that the mass of the fifth physical state is around 520 $\mathrm{MeV}$, which could be identified as $\eta(548)$. In this case $\eta(548)$ turns out to be a mixture of $u \bar{u}+d \bar{d}$ 
Table 5. The numerical analysis of the $5 \times 5$ mixing in the $0^{-+}$channel. Here we have used $\eta^{\prime}(958), \eta(1275)$, $\eta(1405), \eta(1475)$, and $R\left(\eta(1475) / \eta^{\prime}(958)\right)=0.95$ as the input. The fifth state could be interpreted as $\eta(548)$.

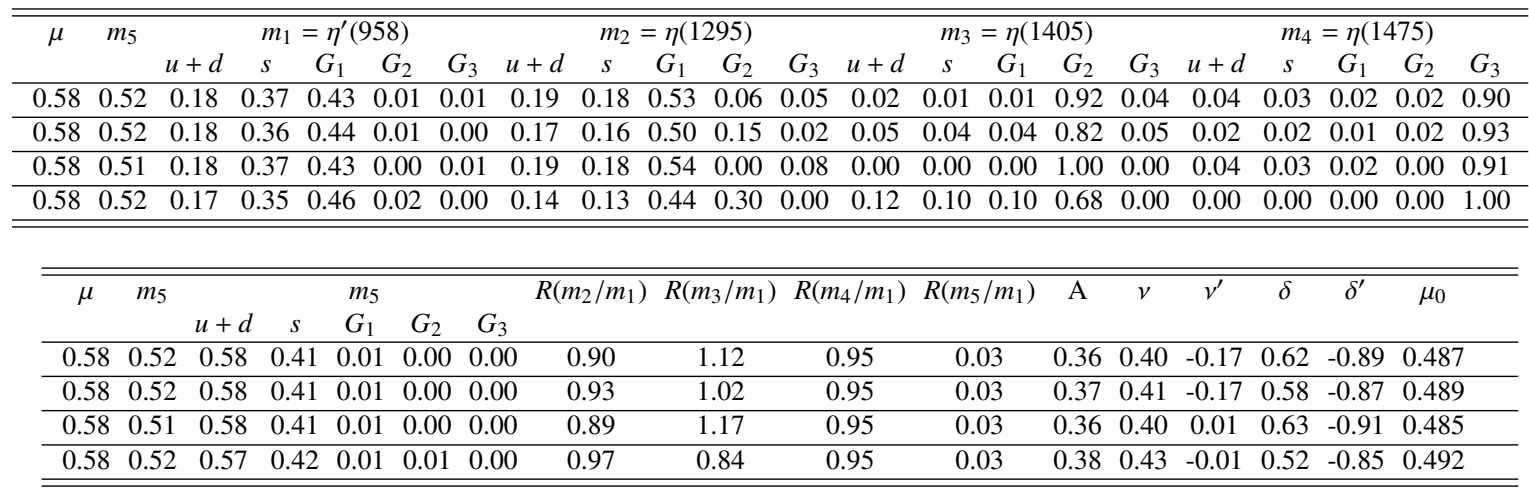

Table 6. The numerical analysis of the $3 \times 3$ mixing in the $0^{-+}$channel. Here we choose $\eta^{\prime}(958)$ and $\eta(1405)$ as the input and vary the mass of $\eta(548)$ to obtain the table. No solution can be found when $m(\eta(548))>541 \mathrm{MeV}$.

\begin{tabular}{ccccccccccccccccc}
\hline \hline$m(\eta(548))$ & $A$ & $v$ & $\mu$ & \multicolumn{4}{c}{$m_{1}=\eta(548)$} & \multicolumn{3}{c}{$m_{2}=\eta^{\prime}(958)$} & \multicolumn{3}{c}{$m_{3}=\eta(1405)$} & $R\left(m_{3} / m_{2}\right)$ & $R\left(m_{1} / m_{2}\right)$ \\
& & & & $u+d$ & $s$ & $G$ & $u+d$ & $s$ & $G$ & $u+d$ & $s$ & $G$ & \\
\hline 510 & 0.34 & 0.51 & 0.64 & 0.60 & 0.39 & 0.01 & 0.23 & 0.47 & 0.30 & 0.17 & 0.14 & 0.69 & 1.2 & 0.05 \\
\hline 520 & 0.41 & 0.55 & 0.60 & 0.56 & 0.43 & 0.01 & 0.16 & 0.33 & 0.51 & 0.28 & 0.24 & 0.48 & 0.49 & 0.03 \\
\hline 530 & 0.50 & 0.48 & 0.55 & 0.51 & 0.48 & 0.01 & 0.09 & 0.19 & 0.72 & 0.40 & 0.34 & 0.27 & 0.19 & 0.02 \\
\hline 540 & 0.58 & 0.22 & 0.49 & 0.47 & 0.53 & 0.00 & 0.01 & 0.03 & 0.95 & 0.52 & 0.43 & 0.04 & 0.02 & 0.003 \\
\hline \hline
\end{tabular}

and $s \bar{s}$, while $\eta^{\prime}(958)$ becomes largely a mixture of $s \bar{s}$ and a $g g$ chromoball, with less than $20 \%$ contamination of $u \bar{u}+d \bar{d}$. And $\eta(1295)$ is made of more than $50 \% g g$ chromoball and less than $20 \%$ $u \bar{u}+d \bar{d}$ and $s \bar{s}$ each. But remarkably, the table shows that $\eta(1405)$ and $\eta(1475)$ are mainly the $g g$ and $g g g$ chromoball states. Moreover, the $J / \psi$ radiative decay ratios $R\left(\eta(1405) / \eta^{\prime}(958)\right)$ is perfect, although $R\left(\eta^{\prime}(958) / \eta(548)\right)$ looks a bit larger. This looks interesting and reasonable.

Notice that $\delta^{\prime}$ in the table turn out to be negative, which tells that the mass of the three chromon bound state is smaller than the sum of the chromon mass. This implies that the binding of three chromons is quite strong. So in the table we have estimated the chromon mass $\mu_{0}$ of the three chromons state, with $G_{3}=9 \mu_{0}^{2}$.

Of course, we have different views in the literature. The popular view that PDG endorses is that $\eta(548)$ and $\eta^{\prime}(958)$ are predominantly the $u \bar{u}+d \bar{d}$ and $s \bar{s}$ states, and that $\eta(1295)$ and $\eta(1475)$ are the first radial excitations of $\eta(548)$ and $\eta^{\prime}(958)[8,9]$. But it is widely agreed that $\eta(1405)$ is indeed a pseudo-scalar glueball $[8,9]$. This is endorsed by PDG and by our analysis, although there exists a lattice result which might contradict with this view [6].

Our result implies that the spectrum of the light pseudo-scalar mesons could be understood within the context of the quarkonium-chromoball mixing.

To test this quark and chromon model further, we now consider the $3 \times 3$ mixing with only three physical states, $\eta(548), \eta^{\prime}(958)$, and $\eta(1405)$, excluding the supposedly radially excited states $\eta(1295)$ and $\eta(1475)[8,9]$. 
Normally in the $3 \times 3$ mixing we could use the three masses and (13) as the input to diagonalize the mass matrix, but in this case we could not find the solution. So we choose only two mass eigenvalues, $\eta^{\prime}(958)$ and $\eta(1405)$, and vary the mass of $\eta(548)$. With this we obtain Table 6.

Interestingly, when the mass of $\eta(548)$ becomes $510 \mathrm{MeV}$, the radiative decay ratio $R(\eta(1405) / \eta(958)) \simeq 1.2$ becomes close to the experimental value 0.95 . In this case $\eta(548)$ becomes $60 \% u \bar{u}+d \bar{d}$ and $39 \% s \bar{s}$, but $\eta^{\prime}(958)$ becomes a mixture of $47 \% s \bar{s}$ and $30 \% g g$. And $\eta(1405)$ becomes predominantly $(69 \%)$ a chromoball.

To understand the physical meaning of Table 6, we notice that the physical contents depends very much on the mass of $\eta(548)$. Moreover, as the mass approaches to the physical value $548 \mathrm{MeV}$, $\eta^{\prime}(958)$ becomes predominantly the glueball. This is troublesome, and does not seem to support the PDG view that $\eta(1295)$ and $\eta(1475)$ are the radial excitations of $\eta(548)$ and $\eta^{\prime}(958)$ at all. This implies that our result shown in Table 5 is at least as good as the PDG view, although this matter has to be studied more carefully.

In this section we have discussed the numerical analysis of the quarkonium-chromoball mixing within the context of the quark and chromon model in three channels $0^{++}, 2^{++}$, and $0^{-+}$below $2 \mathrm{GeV}$. Although the results seem to work, the numerical results are still inconclusive.

The reason is that theoretically it must be clear that the numerical mixing should be regarded as an approximation. Moreover, technically the equation we need to solve to diagonalize the mass matrix is very rigid and sensitive to the ad hoc constraints we have imposed. With these shortcomings it is natural that our results are not perfect. Nevertheless, it is fair to say that the above mixing analysis does show that the quark and chromon model provides a conceptually simple way to understand the glueballs and their mixing with quarkonia.

\section{Discussions}

In this paper we have discussed the hadron spectrum of the ECD obtained from the Abelian decomposition of QCD. Although ECD is mathematically identical to QCD, it makes the hidden dynamical structures of QCD explicit. In particular, it assures the existence of two types of gluons and generalizes the quark model to the quark and chromon model.

The quark and chromon model has clear advantages over other glueball models. The bag model has no constituent gluon since the bag is made of the gluon field, so that it is very difficult to figure out the glueball spectrum. The constituent model has the constituent gluons, but has no the binding gluons to bind them.

The quark and chromon model resolves this difficulty and provides a new picture of glueballs made of chromons. Moreover, the quark and chromon model predicts new hybrid hadronic states made of quarks and chromons. This is made possible by the Abelian decomposition.

The Abelian decomposition tells that there are two types of gluons which play different roles, and decomposes QCD to the restricted part which describes the core dynamics of QCD and the valence part which represents the colored source of QCD. This simplifies the QCD dynamics greatly, and provides the clear picture of the glueballs and hybrid hadrons. Moreover, this provides a clear picture of the glueball-quarkonium mixing.

In particular, this allows us to demonstrate the monopole condensation, more precisely the monopole-antimonopole pair condensation $[15,16]$. We can verify this monopole condensation ex- 
perimentally by searching for the monoball, the $0^{++}$vacuum flucuation of the monopole condensation. The monoball, if exist, could have mass around $\Lambda_{Q C D}$. This implies that $f_{0}(500)$ could be the monoball candidate. Of course, at the moment it is not clear if this is the case. But the search for the monoball should be treated as one of the most important issue in QCD, because this could confirm the monopole condensation in QCD.

Moreover, the Abelian decomposition can be tested directly by experiment. Two types of gluon mean two types of gluon jets, the neuron jet and the chromon jet. The neuron jet might look like the photon jet, since the neurons are color neutral. But the chromon jet should liook like the quark jet, since the chromons are colored. So we can test the Abelian decomposition experimentally confirming the existence of two different gluon jets.

The main purpose of this paper was to discuss the quark and chromon model and provide the general framework of the glueball-quarkonium mixing mechanism in this model. Although the numerical result is not completelt satisfactory, it does show that the model has clear advantage over other models. A more complete numerical mixing analysis will be provided in a separate publication [23].

\section{Acknowledgment}

The work is supported in part by the National Research Foundation of (NRF) funded by funded by the Ministry of Education (Grant 2015-R1D1A1A0-1057578) and by Center for Quantum Space-time at Sogang University.

\section{References}

[1] H. Fritzsch and P. Minkowski, Nuovo Cimento 30A, 393 (1975); P. G. O. Freund and Y. Nambu, Phys. Rev. Lett. 34, 1645 (1975).

[2] J. Kogut, D. Sinclair, and L. Susskind, Nucl. Phys. B114, 199 (1976).

[3] R. L. Jaffe and K. Johnson, Phys. Lett. B60, 201(1976); P. Roy and T. Walsh, Phys. Lett. B78, 62 (1978).

[4] J. Coyne, P. Fishbane, and S. Meshkov, Phys. Lett B91, 259 (1980); M. Chanowitz, Phys. Rev. Lett. 46, 981 (1981); J. Cornwall and A. Soni, Phys. Lett. B120, 431 (1983).

[5] G. Bali et al., Phys. Lett. B309, 378 (1993); C. Morningstar and M. Peardon, Phys. Rev. D60, 034509 (1999).

[6] W. Lee and D. Weingarten, Phys. Rev. D61, 014015 (2000); Y. Chen et al., Phys. Rev. D73, 014516 (2006).

[7] K. Olive et al., [Particle Data Group], Review of Particle Physics, Chin. Phys. 38, 090001 (2014).

[8] C. Amsler and N. Tornqvist, Phys. Rep. 389, 61 (2004); V. Mathieu, N. Kochelev, and V. Vento, Int. J. Mod. Phys. E18, 1 (2009).

[9] W. Ochs, J. Phys. G40, 043001 (2013).

[10] J. Dudek et al., Eur. Phys. J. A48, 187 (2012).

[11] Y. M. Cho, Phys. Rev. D21, 1080 (1980).

[12] Y. M. Cho, Phys. Rev. Lett. 46, 302 (1981); Phys. Rev. D23, 2415 (1981); Y. S. Duan and M. L. Ge, Sci. Sinica 11,1072 (1979).

[13] S. Kato, K. Kondo, T. Murakami, A. Shibata, T. Shinohara, and S. Ito, Phys. Lett. B632, 326 (2006); S. Ito, S. Kato, K. Kondo, T. Murakami, A. Shibata, and T. Shinohara, Phys. Lett. B645, 67 (2007). 
[14] N. Cundy, Y. M. Cho, W. Lee, and J. Leem, Phys. Lett. B729, 192 (2014); Nucl. Phys. B, in press.

[15] Y. M. Cho, Franklin H. Cho, and J. H. Yoon, Phys. Rev. D87, 085025 (2013).

[16] Y. M. Cho, Int. J. Mod. Phys. A29, 1450013 (2014).

[17] Y. M. Cho, X. Y. Pham, Pengming Zhang, Ju-Jun Xie, and Li-Ping Zou, Phys. Rev. D91, 114020 (2015).

[18] J. Schwinger, Phys. Rev. 82, 664 (1951).

[19] Y. M. Cho and D. G. Pak, Phys. Rev. Lett. 86, 1947 (2001); 91, 039151.

[20] D. Gross anf F. Wilczek, Phys. Rev. Lett. 30, 1343 (1973); H. Politzer, Phys. Rev. Lett. 30, 1346 (1973).

[21] V. Schanbacher, Phys. Rev. D26, 489 (1982).

[22] Y. M. Cho, M. L. Walker, and D. G. Pak, JHEP 04, 073 (2004).

[23] Pengming Zhang, Ju-Jun Xie, Li-Ping Zou, and Y. M. Cho, arXiv, hep-ph/1606.02374 (2016), to be published. 